\title{
Detection of submucosal gastric fundal varices with multi- detector row CT angiography
}

\author{
J K Willmann, D Weishaupt, T Böhm, T Pfammatter, B Seifert, B Marincek, \\ P Bauerfeind
}

Gut 2003;52:886-892

\begin{abstract}
Background and aim: The diagnosis of submucosal fundal varices is challenging. Currently, endoscopy and endoscopic ultrasound (EUS) are considered most useful for this purpose. The aim of this study was to evaluate if multi-detector row CT (MDCT) angiography contributes to the diagnosis of submucosal fundal varices.

Patients and methods: Twenty two patients with endoscopically suspected fundal varices were prospectively included in the study. All patients underwent EUS and MDCT angiography. Levels of agreement between EUS and MDCT angiography for the detection of submucosal and perigastric fundal varices were evaluated by three blinded independent readers. In addition, variceal size and location, as well as afferent and efferent vessels of the submucosal varices, were determined. Results: Good or excellent image quality of MDCT angiography was obtained in 21/22 patients $(95 \%)$. Based on EUS, submucosal varices were detected in 16 of 22 patients $(73 \%)$ and perigastric varices in $22 / 22$ patients (100\%). Using MDCT angiography, the presence of submucosal varices was confirmed in all of these 16 patients by all three readers. Perigastric varices were also confirmed in all 22 patients by all three readers. In addition, all three readers noted the presence of a submucosal varix in an additional patient which was not detected on initial EUS. MDCT angiography showed an excellent interobserver reliability with regard to variceal diameter $(\kappa=0.90)$ and variceal location $(\kappa=0.94)$. Based on MDCT angiography, afferent and efferent vessels of submucosal varices included the left gastric vein in 11 (65\%), the posterior/short gastric veins in 15 (88\%), gastrorenal shunts in 10 (59\%), the left inferior phrenic vein in six $(35 \%)$, and the left pericardiophrenic vein in six $(35 \%)$ of 17 patients. Conclusions: MDCT angiography is equivalent to EUS in terms of detection and characterisation of fundal varices, in particular with regard to the distinction between submucosal and perigastric fundal varices.
\end{abstract}

See end of article for authors' affiliations

Correspondence to: Dr D Weishaupt, Institute of Diagnostic Radiology, University Hospital, Rämistrasse 100, 8091 Zurich, Switzerland: dominik.weishaupt@ dmr.usz.ch Accepted for publication 2 December 2002

$\mathrm{F}$ undal varices have been increasingly recognised as a major cause of gastrointestinal bleeding in patients with portal hypertension. ${ }^{1}$ Compared with oesophageal variceal bleeding, haemorrhage caused by fundal varices is frequently more severe and haemostatic control is reported to be more difficult. ${ }^{12}$

The afferent vessels of fundal varices include the left gastric, short gastric, and posterior gastric veins. ${ }^{3}{ }^{4}$ The efferent veins drain through oesophageal veins, the left inferior phrenic vein, the left pericardiacophrenic vein, or the left renal vein into the vena cava (fig 1). ${ }^{3}{ }^{4}$ According to their location with regard to the gastric fundal wall, fundal varices are divided into submucosal fundal varices and perigastric (adventitial) fundal varices (fig 1). ${ }^{35-7}$ The distinction between submucosal and perigastric varices is of major clinical interest as fundal variceal bleeding is almost always caused by submucosal fundal varices. ${ }^{189}$ In addition, submucosal fundal varices can be treated successfully by endoscopic therapy.

The diagnosis of submucosal fundal varices is challenging. Oesophagogastroduodenoscopy is usually the initial investigation in patients with suspected fundal varices although the distinction between fundal varices and gastric folds, particularly in patients with hypertensive gastropathy, may be difficult. ${ }^{57810}$ Currently, endoscopic ultrasound (EUS) is considered most useful in the evaluation and diagnosis of submucosal fundal varices. ${ }^{57810}$ EUS allows visualisation of the different layers of the gastric wall and permits differentiation between submucosal and perigastric fundal varices. ${ }^{57810}$ However, EUS is invasive, not widely available, and examiner dependent. Hence a non- invasive imaging modality would be preferable in these high risk patients.
The role of splenoportography or percutaneous transhepatic portography, magnetic resonance (MR), and computed tomography (CT) imaging is limited in the distinction between submucosal and perigastric fundal varices, although both modalities allow entire assessment of the portal venous system and its collaterals. ${ }^{911-14}$

Multi-detector row CT (MDCT) angiography is gaining increasing acceptance as a minimally invasive technique for imaging the abdominal vascular system. ${ }^{15-17}$ MDCT angiography allows visualisation of small visceral vessels by offering shorter acquisition times, less motion artefacts, and increased spatial resolution compared with single detector helical CT. ${ }^{15}$ These characteristics may be useful in order to assess the gastric fundus for the presence and differentiation of submucosal and perigastric varices.

The purpose of this study was to assess feasibility, accuracy, and reliability of MDCT angiography in the detection of fundal varices with particular focus on its ability to differentiate between submucosal and perigastric fundal varices in patients with portal hypertension or splenic vein occlusion. Findings were compared with EUS.

Abbreviations: EUS, endoscopic ultrasound; MR, magnetic resonance; $\mathrm{CT}$, computed tomography; MDCT, multi-detector row CT; MIPs, maximum intensity projections; VRs, volume renderings; MPRs, multiplanar reconstructions; TIPSS, transjugular intrahepatic portosystemic shunt stent. 


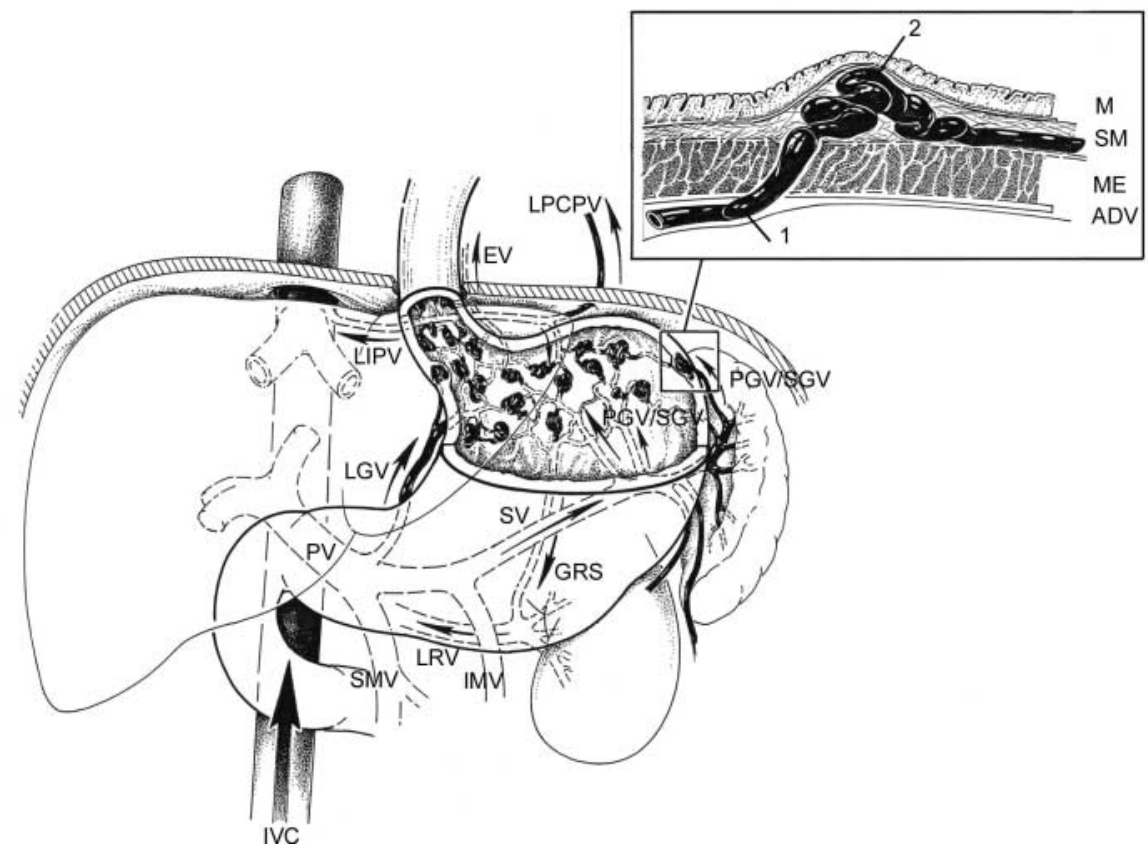

Figure 1 Schematic diagram showing the upper abdominal organs from a ventral view with emphasis on the venous anatomy and the afferent and efferent veins of gastric fundal varices in portal-venous hypertension. The afferent veins of fundal varices include the left gastric vein (LGV) and the posterior/short gastric veins (PGV/SGV). The efferent veins drain through oesophageal veins (EV), the left inferior phrenic vein (LIPV), the left pericardiacophrenic vein (LPCPV), and the gastrorenal shunts (GRS). PV, portal vein; SV, splenic vein; LRV, left renal vein; SMV, superior mesenteric vein; IMV, inferior mesenteric vein; IVC, inferior vena cava. Schematic representation (box) of the wall structures of the gastric fundal wall demonstrates a perigastric (adventitial) varix (1) located within the adventitial layer at the exterior border of the gastric wall. The perigastric (adventitial) varix penetrates the muscularis externa (ME) and forms a submucosal varix (2). $M$, mucosa; SM, submucosa; ADV, adventitia/serosa.

\section{MATERIALS AND METHODS}

\section{Study design and patients}

Between October 2000 and January 2002, 22 patients (16 males, six females; mean age 51 years (range 21-74)) with endoscopic suspicion of fundal varices admitted to our institution were considered eligible for enrolment in this study. Suspicion of fundal varices was established according to the following criteria: (1) suspicion of fundal varices at oesophagogastroduodenoscopy in patients with portal hypertension or splenic vein occlusion and with a history of previous ( $>1$ month) or recent $(<1$ month) upper gastrointestinal bleeding; (2) patients with known portal hypertension or splenic vein occlusion and an episode of fundal variceal bleeding, as established by emergency oesophagogastroduodenoscopy with subsequent direct endoscopic therapy who underwent routine oesophagogastroduodenoscopy as part of the follow up programme for evaluation of the effect of endoscopic therapy. Exclusion criteria included a history of renal insufficiency and adverse reactions to iodinated contrast agents or refusal of the study protocol.

All patients included in this study underwent EUS and MDCT angiography within one week after the initial oesophagogastroduodenoscopy. The study was approved by the Hospital Institutional Review Board and written informed consent was obtained from all patients.

\section{MDCT angiography}

All MDCT angiograms were performed on a four channel MDCT scanner (SOMATOM Volume Zoom; Siemens AG, Forchheim, Germany). Immediately prior to scanning, 500$750 \mathrm{ml}$ of plain water were administered orally to all patients. Water was used as a negative contrast agent to distend the stomach and improve visualisation of the gastric wall. To standardise distention of the stomach, all patients in this study were asked to drink the water immediately prior to scanning while sitting on the table of the CT scanner. Neither anticholinergic agents nor glucagon were administered to any patient. The scanning range was planned starting from the distal third of the oesophagus and ending at the lower pole of the kidneys to encompass the whole extent of the stomach and to include the portal vein and its tributaries in the upper abdomen.

MDCT scanning was performed during inspiratory breathhold following administration of $120 \mathrm{ml}$ of a non-ionic iodinated contrast medium (Ultravist 300; Schering, Berlin, Germany; $300 \mathrm{mg}$ iodine $/ \mathrm{ml}$ ). The contrast agent was injected at a flow rate of $3 \mathrm{ml} / \mathrm{s}$ using an automated injector (Ulrich Medical AG, Ulm-Jungingen, Germany). Scanning was started 65 seconds later during the portal-venous phase of the contrast medium. Technical parameters for the MDCT examinations were as follows: beam collimation $4 \times 1 \mathrm{~mm}$; table feed $5 \mathrm{~mm}$ per rotation (pitch 1.25); and rotation time $0.5 \mathrm{~s}$. Image reconstruction was performed with a slice thickness of 1.25 $\mathrm{mm}$ at an interval of $0.6 \mathrm{~mm}$. MDCT scanning was performed in the supine position at $180 \mathrm{~mA}$ and $120 \mathrm{kV}$ with a standard algorithm and a $512 \times 512$ matrix size.

\section{Image analysis}

All further data processing and interpretation were performed on an Advantage Windows 4.0 workstation (GE Medical Systems, Milwaukee, Wisconsin, USA) equipped with a software tool which allowed generation of maximum intensity projections (MIPs), volume renderings (VRs), and multiplanar reconstructions (MPRs). One radiologist experienced in three dimensional reconstruction techniques processed all MDCT angiograms into MIPs, VRs, and MPRs. This radiologist was not involved in the following image analysis and was blinded to the patient's data and results of EUS. All three dimensional reconstructions were stored on the hard disk memory of the workstation for subsequent image analysis. 
Table 1 Subjective impression of image quality of the gastric fundus on multi-detector row CT angiograms, as assigned by the three readers

\begin{tabular}{rlrrrrr}
\hline & \multicolumn{4}{l}{ Subjective impression of image quality of gastric fundus (grade) } & \\
\cline { 2 - 5 } & 1 & 2 & 3 & 4 & 5 & Total \\
\hline Reader & 0 & 0 & 1 & 11 & 10 & 22 \\
No 1 & 0 & 0 & 1 & 7 & 14 & 22 \\
No 2 & 0 & 0 & 2 & 12 & 8 & 22 \\
No 3 & 0 & & & & \\
\hline
\end{tabular}

*Grade 1, unacceptable (diagnostic information not obtained); grade 2, poor (poor differentiation of details of the gastric fundus including gastric fundus lumen, gastric fundus wall, and perigastric space); grade 3, fair (fair differentiation of details of the gastric fundus); grade 4, good (good differentiation of details of the gastric fundus); and grade 5, excellent image quality (excellent differentiation of details of the gastric fundus).

Three independent radiologists experienced in cross sectional imaging (reader Nos 1, 2 and 3) performed image analysis separately and in random order on the basis of transverse source images, MIPs, VRs, and MPRs. All three readers were blinded to patient data, including clinical history, clinical findings, and results of EUS.

The presence of varices located in the fundus of the stomach was assessed by both readers. According to the classification of gastric varices as proposed by Sarin and Kumar, ${ }^{18}$ varices termed fundal varices in this study included either gastro-oesophageal varices that extend to the gastric fundus (so-called gastrooesophageal varices type 2 according to Sarin and $\mathrm{Kumar}^{18}$ ) or isolated ectopic fundal varices (so-called isolated gastric varices type 1 according to Sarin and $\mathrm{Kumar}^{18}$ ). When fundal varices were assessed as present, varices were classified as submucosal or perigastric. ${ }^{3-7}$ Submucosal fundal varices were defined as varices located within the wall of the gastric fundus which demonstrated contrast enhancement during the portal-venous phase. ${ }^{79}$ Perigastric (adventitial) fundal varices were defined as serpiginous vascular structures demonstrating contrast enhancement during the portal-venous phase which were located along the outside border of the gastric fundal wall. ${ }^{30}$ Each reader had to indicate his diagnostic confidence in the presence or absence of submucosal or perigastric varices using a five point Likert scale, with the following scores: 1 , submucosal/perigastric varices definitely absent; 2, submucosal/perigastric varices probably absent; 3; submucosal/perigastric varices possibly present (indeterminate); 4, submucosal/perigastric varices probably present; 5 , submucosal/perigastric varices definitely present. When submucosal varices were considered to be present (score 4 or 5 ), each of the three readers was asked to measure the diameter of the largest visible submucosal varix and to classify the varix as a small $(<5 \mathrm{~mm}$ in diameter) or large $(\geqslant 5 \mathrm{~mm})$ submucosal varix. ${ }^{52}$ Moreover, the readers were asked to assign the site of the submucosal varices using the following terms: 1 , location of the submucosal fundal varices at the medial border; 2, at the lateral border; and 3, at the medial and lateral border of the fundus.

The afferent and efferent vessels of submucosal varices as well as the presence or absence of the portal vein and splenic vein thrombosis or oesophageal varices were assessed by all three readers in consensus. This image evaluation was performed with a three week delay following separate reading sessions. As it is sometimes difficult to differentiate between the posterior gastric vein and the short gastric vein, we called these collaterals posterior/short gastric vein..$^{22}$

By using a commercially available PC program (WinDose, version 2.la; Scanditronix- Wellhöfer Dosimetrie, Schwarzenbruck, Germany) the effective dose of MDCT angiography for a mean cranio-caudal scanning range of $21 \mathrm{~cm}$ and a tube current of $180 \mathrm{~mA}$ at $120 \mathrm{kV}$ was estimated to be $5.0 \mathrm{mSv}$ for women and $4.0 \mathrm{mSv}$ for men.

\section{Endoscopic ultrasound (EUS)}

All patients with clinical suspicion of fundal varices underwent EUS performed by one experienced gastroenterologist.
EUS was performed using a radial scanning echoendoscope GF-UM20 (Olympus, Japan) at 7.5 and $12 \mathrm{MHz}$ as well as an echoendoscope (FG-38UX; Pentax, Japan) with longitudinal scanning and equipped with a $7.5 \mathrm{MHz}$ transducer and Doppler mode. EUS was performed under intravenous sedation using midazolam $(2.5-5 \mathrm{mg})$ and/or pethidine (25-50 mg). All studies were documented on videotape for subsequent review. The gastroenterologist interpreting the EUS was unaware of the MDCT angiographic findings. On EUS, submucosal and perigastric varices were defined as hypoechoic vessels within the wall of the gastric fundus or in the tissue and spaces exterior to the adventitia of the gastric fundus, respectively, with continuous colour Doppler flow in the vascular lumen. ${ }^{20}$ For determination of the presence of submucosal and perigastric varices, size and location of submucosal fundal varices, as well as classification of submucosal and perigastric fundal varices, the endoscopist used the same grading scales as used for MDCT angiograms. To avoid erroneous measurements of variceal diameter due to tangential imaging, vessels were measured at a point where their cross section appeared circular. ${ }^{20}$

\section{Statistical analysis}

Agreement between MDCT angiography and EUS with regard to the diagnosis of submucosal and perigastric varices as well as for classification of variceal size and determination of variceal location are given as percentages. Interobserver agreement between all three readers in terms of classification of variceal size and location of submucosal fundal varices on MDCT angiographic images was determined by calculating kappa values $(\kappa) .{ }^{23}$ As there is no definitive standard of reference for the presence of submucosal fundal varices, we did not calculate sensitivity, specificity, positive and negative predictive values, or accuracy of MDCT angiography for this purpose.

Table 2 Diagnostic confidence with regard to the presence of submucosal fundal varices in 22 patients using multi-detector row $\mathrm{CT}$ (MDCT) angiography and endoscopic ultrasound (EUS)

\begin{tabular}{lcllll}
\hline & \multicolumn{3}{l}{ MDCT angiography } & \\
\cline { 2 - 4 } $\begin{array}{l}\text { Diagnostic } \\
\text { confidence }\end{array}$ & $\begin{array}{l}\text { Reader No 1 } \\
\text { (n) }\end{array}$ & $\begin{array}{l}\text { Reader No 2 } \\
\text { (n) }\end{array}$ & $\begin{array}{l}\text { Reader No 3 } \\
\text { (n) }\end{array}$ & $\begin{array}{l}\text { EUS } \\
\text { (n) }\end{array}$ \\
\hline Score 1 & 4 & 4 & 3 & 5 \\
Score 2 & 1 & 1 & 2 & 1 \\
Score 3 & 0 & 0 & 0 & 0 \\
Score 4 & 2 & 1 & 1 & 1 \\
Score 5 & 15 & 16 & 16 & 15 \\
\hline
\end{tabular}

Score 1, submucosal varix definitely absent; 2 , submucosal varix probably absent; 3 ; submucosal varix possibly present (indetermin ate); 4 , submucosal varix probably present; 5 , submucosal varix definitely present. $\mathrm{n}=$ number of patients. 

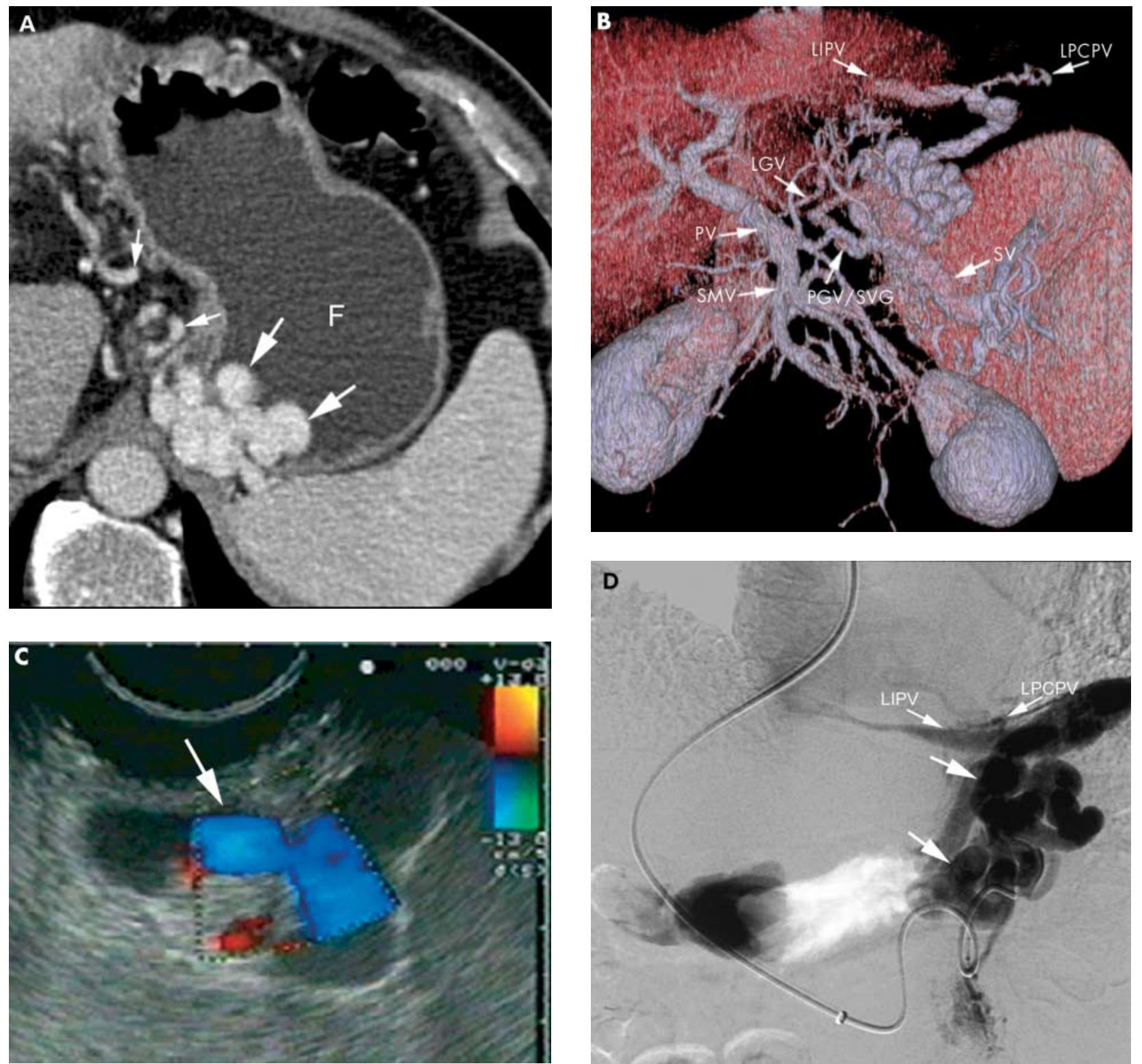

Figure 2 Submucosal and perigastric fundal varices in a 68 year old male with alcohol related liver cirrhosis Child-Pugh class A. (A) Transverse source image of multi-detector row CT (MDCT) angiography during the portal-venous phase shows a conglomerate of submucosal varices $\geqslant 5 \mathrm{~mm}$ in diameter (large white arrows) located on both medial and lateral borders of the water filled gastric fundus (F). Perigastric varices (small white arrows) are also noted. (B) Volume rendering of MDCT angiographic data set during the portal- venous phase demonstrates the submucosal fundal varices with afferent vessels, including the left gastric vein (LGV) and the posterior/short gastric veins (PGV/SGV). The efferent veins drain through the left inferior phrenic vein (LIPV) and the left pericardiacophrenic vein (LPCPV). PV, portal vein SV, splenic vein; SMV, superior mesenteric vein. (C) Corresponding endoscopic ultrasound image of the same patient confirms the presence of submucosal fundal varices, visible as large hypoechoic vessels (arrow) with continuous colour Doppler flow within the wall. (D) Direct selective digital subtraction venogram of the posterior/short gastric vein after transjugular intrahepatic portosystemic shunt stent placement in the same patient demonstrates a conglomerate of fundal varices (arrows) with the efferent vessels draining through the left inferior phrenic (LIPV) and the left pericardiophrenic veins (LPCPV).

The results of the consensus reading for determination of afferent and efferent veins of fundal varices were compared with splenoportography, where possible, when it was performed during the study period for clinical reasons.

\section{RESULTS}

\section{Patient characteristics}

A history of a previous or recent episode of upper gastrointestinal bleeding from oesophageal varices was present in $10 / 22$ patients $(45 \%)$. Six of 22 patients $(27 \%)$ had a history of an endoscopically documented previous or recent fundal variceal bleed. In each of these six patients, endoscopic guided sclerotherapy was performed prior to inclusion in the study (mean 87 days (range 2-276)). During the study period, one of 22 patients $(5 \%)$ died eight weeks after EUS and MDCT angiographic examination. Autopsy was performed on this patient and revealed the presence of perigastric and submucosal fundal varices, as demonstrated by EUS and MDCT angiography. Four of 22 patients (18\%) underwent transjugular intrahepatic portosystemic shunt stent (TIPSS) therapy during the study period. Splenoportography was performed in these four patients.

\section{Imaging findings}

Contrast enhanced MDCT angiography was well tolerated, with no complications, by all 22 patients. No sedation or analgesia was necessary in any patient. 

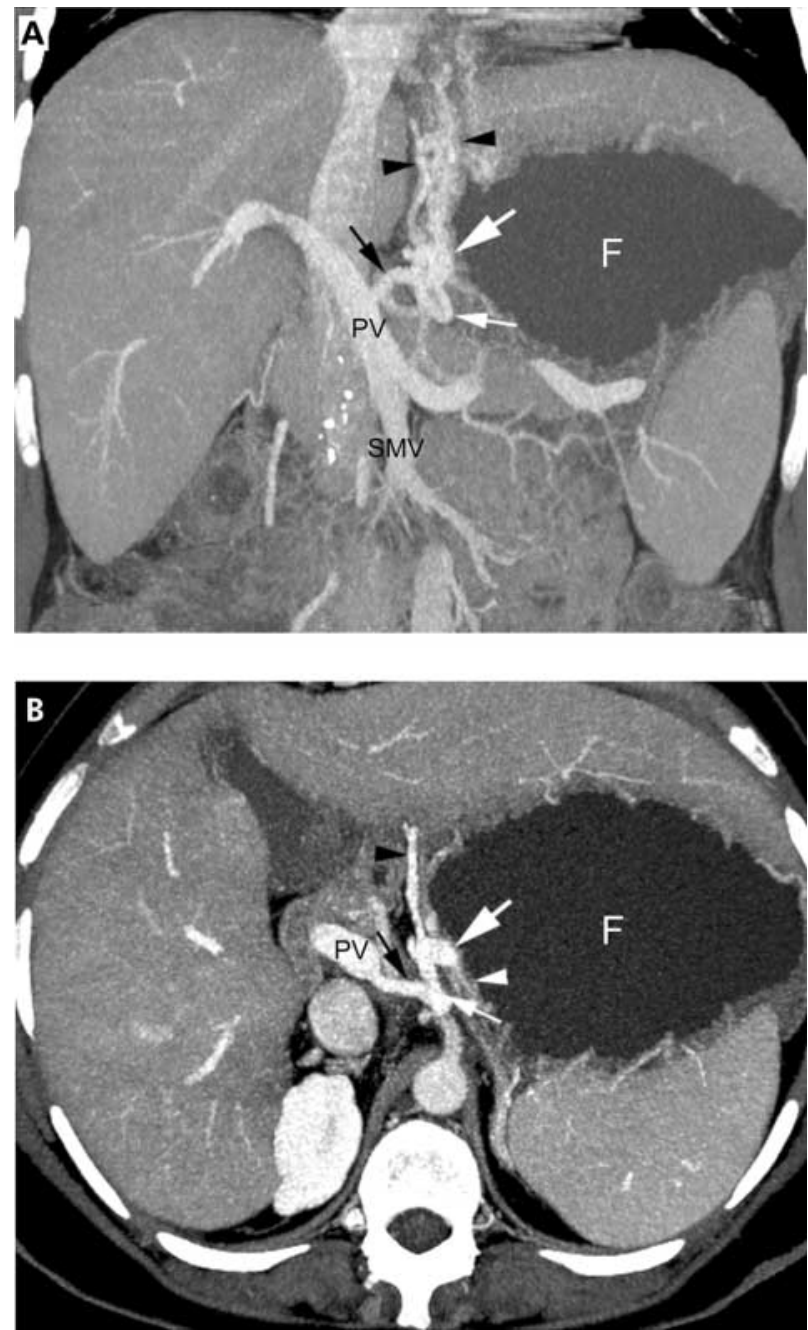

Figure 3 A 31 year old female with portal hypertension due to hepatitis $C$ induced liver cirrhosis Child Pugh class $A$ and the presence of submucosal and perigastric fundal varices. (A) Coronal thin slap maximum intensity projection of multi-detector row CT (MDCT) angiography during the portal-venous phase shows a large submucosal varix ( $\geqslant 5 \mathrm{~mm}$ in diameter) (large white arrow) located on the medial border of the water filled gastric fundus (F). A

perigastric varix (small white arrow) is also noted. The afferent vesse includes the left gastric vein (black arrow) and efferent vessels drain through the oesophageal veins (black arrowheads). PV, portal vein; SMV, superior mesenteric vein. (B) The submucosal location within the gastric wall (white arrowhead) of the varix (large white arrow) is more visible on the transverse thin slap maximum intensity projection.

Table 1 summarises the image quality of the gastric fundus on MDCT angiograms, as assigned by the readers. Good or excellent image quality (grades 4 and 5 ) was assigned in 21/22 patients $(95 \%)$ by reader No 1 and reader No 2, and in 20 patients $(91 \%)$ by reader No 3 . None of the MDCT angiograms was graded as poor or unacceptable image quality (grades 2 or 1).

Table 2 shows diagnostic confidence, as reported by EUS as well as by MDCT angiography, with regard to the presence or absence of submucosal fundal varices (figs 2, 3). All three readers reported submucosal fundal varices in 17 patients (77\%) with confidence scores of 4 and 5 (probably present or definitely present). Definite presence of submucosal varices was noted by all readers in all of the six patients with a history of documented fundal variceal bleeding who underwent endoscopically guided sclerotherapy of their submucosal fundal varices prior to inclusion (fig 4). On EUS, submucosal varices were also diagnosed as probably or definitely present in one and five patients, respectively. All three readers disagreed

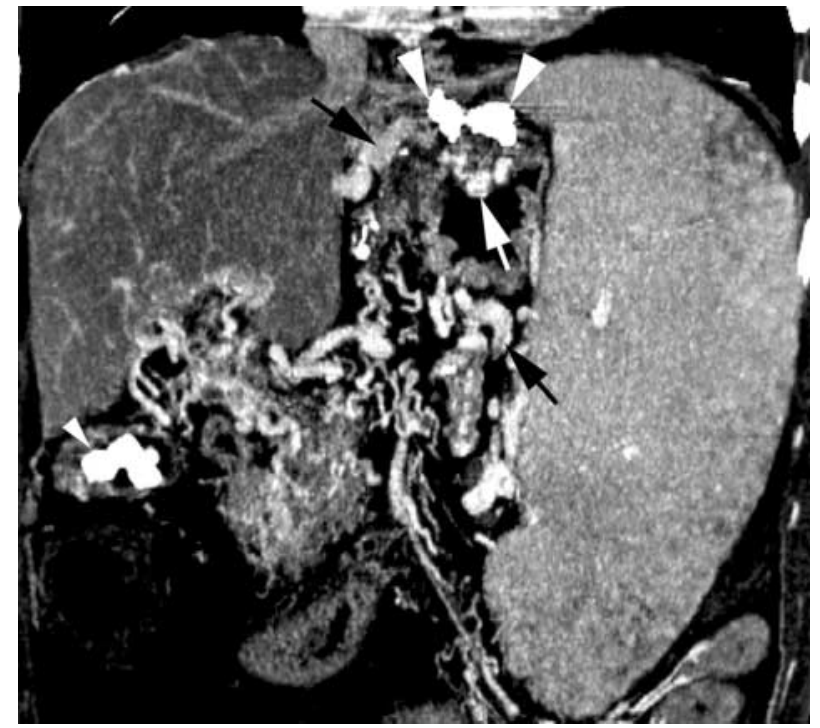

Figure 4 Recurrent submucosal fundal varix in a 60 year old female with chronic thrombosis and cavernous transformation of the portal vein and a history of recent fundal variceal bleeding with subsequent sclerosing therapy. Coronal multiplanar reconstruction of multi-detector row CT (MDCT) angiographic data set during the portal-venous phase demonstrates a histoacryl deposit in the gastric fundal wall (large arrowheads). A non- sclerosed submucosal fundal varix larger than $5 \mathrm{~mm}$ in diameter (white arrow) as well as perigastric varices (black arrows) are noted. In addition, extensive portal venous collaterals, splenomegaly, and calcified gall stones (small arrowhead) are present.

with the endoscopic findings in one patient $(5 \%)$. In this patient, reader No 1 reported a submucosal fundal varix to be probably present and the two remaining readers to be definitely present. In contrast, on EUS, submucosal varices were considered to be absent in this patient. Repeated EUS, which was performed three weeks later and which was not part of the study protocol, confirmed the presence of a submucosal varix in this patient.

On EUS, definite presence of perigastric varices (score 5) was reported in all 22 patients. A diagnostic confidence score of 5 was also given for all 22 patients by all readers on MDCT angiograms. This resulted in an overall agreement of $100 \%$ (22/22 patients) between both imaging modalities and between all three readers $(\kappa=1.0)$ with regard to the diagnosis and diagnostic confidence of perigastric fundal varices.

When submucosal fundal varices were diagnosed (score 4 or 5) on MDCT angiograms, there was excellent agreement between all readers regarding classification of variceal size $(\kappa=0.90)$. Reader Nos 1 and 3 noted submucosal varices of $\geqslant 5$ $\mathrm{mm}$ in diameter in $13 / 17$ patients $(76 \%)$ and reader No 2 in $14 / 17$ patients $(82 \%)$. On EUS, submucosal varices were classified as $\geqslant 5 \mathrm{~mm}$ in diameter in $12 / 16$ patients $(75 \%)$ and submucosal varices were classified as $<5 \mathrm{~mm}$ in $4 / 16$ patients $(25 \%)$, resulting in an average agreement of $79 \%$ between EUS and MDCT angiography.

Table 3 shows the variceal location in patients having submucosal fundal varices, as seen on MDCT angiography and EUS. Overall, there was excellent agreement $(\kappa=0.94)$ between all three readers evaluating MDCT angiograms with regard to location of submucosal fundal varices. On average, there was $83 \%$ agreement between MDCT angiography and EUS for determination of variceal location.

Table 4 summarises the afferent and efferent vessels of submucosal fundal varices in 17 patients where submucosal fundal varices were present on MDCT angiography. Comparison with splenoportography was possible in all $4 / 22$ patients (18\%) who underwent TIPSS placement during the study period. The afferent and efferent veins, as depicted on MDCT 
Table 3 Location of submucosal varices within the gastric fundus as determined by multi-detector row CT (MDCT) angiography $(n=17)$ and endoscopic ultrasound (EUS) ( $n=16$ )

\begin{tabular}{lcccc}
\hline & \multicolumn{3}{l}{ MDCT angiography } & \\
\cline { 2 - 4 } $\begin{array}{l}\text { Location within the } \\
\text { gastric fundus }\end{array}$ & $\begin{array}{l}\text { Reader } \\
\text { No 1 (n) }\end{array}$ & $\begin{array}{l}\text { Reader } \\
\text { No 2 (n) }\end{array}$ & $\begin{array}{l}\text { Reader } \\
\text { No 3 (n) }\end{array}$ & $\begin{array}{l}\text { EUS } \\
\text { (n) }\end{array}$ \\
\hline $\begin{array}{l}\text { Medial border } \\
\text { Lateral border }\end{array}$ & 5 & 5 & 5 & 3 \\
$\begin{array}{l}\text { Medial and lateral } \\
\text { border }\end{array}$ & 5 & 7 & 6 & 7 \\
Total & 17 & 17 & 6 & 6 \\
\hline n=number of patients. & & & 17 & 16 \\
\hline
\end{tabular}

Table 4 Afferent and efferent vessels of submucosal fundal varices in 17 patients, as assessed by multi-detector row CT (MDCT) angiography

\begin{tabular}{lcc}
\hline & \multicolumn{2}{c}{ MDCT angiography } \\
\cline { 2 - 3 } & $\mathrm{n}$ & $\%$ \\
\hline Left gastric vein & 11 & 65 \\
Posterior/short gastric veins & 15 & 88 \\
Gastrorenal shunt & 10 & 59 \\
Inferior phrenic vein & 6 & 35 \\
Pericardiophrenic vein & 6 & 35 \\
\hline
\end{tabular}

$\mathrm{n}=$ number of patients

angiograms, were confirmed in all four of these patients by splenoportography (fig 2). MDCT angiography detected portal vein thrombosis in $5 / 22$ patients $(23 \%)$, thrombosis of the splenic vein in $3 / 22$ patients $(13 \%)$, and oesophageal varices in $21 / 22$ patients $(95 \%)$.

\section{DISCUSSION}

Development of gastric fundal varices is an important manifestation of portal hypertension. Gastric fundal varices most frequently result from generalised or segmental (portal or splenic vein thrombosis) portal hypertension" and the development of gastric fundal varices may depend on the preexisting venous anatomy in the gastric fundus. The exact incidence of fundal varices is unknown.

As with other portosystemic collaterals anywhere in the abdomen, variceal bleeding is the most life threatening complication of gastric and especially fundal varices, with a mortality rate of up to $30 \%$ at bleeding. ${ }^{24}$ Compared with oesophageal variceal bleeding, haemorrhage from fundal varices is more severe and is associated with significantly higher blood transfusion requirements. ${ }^{24}$ From a clinical point of view, it is essential to recognise that only fundal varices that are located within the gastric wall (that is, submucosal location) may haemorrhage. ${ }^{89}$ In contrast, fundal varices that are located along the outside border of the gastric fundal wall (that is, perigastric/adventitial varices) do not.

The diagnosis of fundal varices, in particular of submucosal fundal varices, is challenging. Using endoscopy, submucosal fundal varices often appear as mass-like nodular and tortuous winding elevations of the mucosa in the fundus. ${ }^{18}$ Due to their deep submucosal location as well as the normal colour and appearance of the overlaying mucosa, it may be difficult to distinguish fundal varices from gastric folds, particularly in the presence of hypertensive gastropathy. ${ }^{581018}$ Moreover, the red colour signs seen on oesophageal varices are rarely seen over fundal varices. ${ }^{18}$ EUS is currently considered the most useful modality for assessment of fundal varices by its ability to demonstrate the different layers of the gastric fundus. This allows for precise information with regard to the presence, size, and exact location of the varix within the gastric fundus (that is, submucosal versus perigastric location of fundal varices)..$^{57810}$ However, a broad use of EUS is hampered by its limited availability in relatively few specialised centres, ${ }^{25}$ its invasiveness, ${ }^{26}$ as well as its operator dependency, with only a fair interobserver agreement with regard to assessment of vascular lesions in the stomach. ${ }^{25}$

On double contrast barium studies, the appearance of fundal varices may be confused with a tumorous lesion. ${ }^{927}$ Splenoportography and direct transhepatic portography allow excellent delineation of the portal and splenic venous system and its collateral circulation. The main limitation of angiography is its inability to determine the relationship of the vessels to the mucosa. ${ }^{914}$

Contrast enhanced conventional and single detector helical CT as well as MR imaging are useful modalities for expeditiously evaluating the overall status of portosystemic vessels in patients with portal hypertension. ${ }^{911-13}$ However, to the best of our knowledge, no attempt has been made to evaluate if CT or MR imaging might provide information on fundal varices, in particular with regard to the relationship between the varix and the mucosa. With the advent of MDCT technology, CT angiography has been improved substantially by offering shorter acquisition times, increased volume coverage, lower dose of contrast medium, and improved spatial resolution for assessing small visceral vessels. ${ }^{15}{ }^{17}$

In this prospective study, we investigated the clinical applicability of MDCT angiography in the diagnosis of submucosal and perigastric fundal varices. The high reliability and robustness of MDCT angiography was reflected in the overall good to excellent image quality and high interobserver agreements between all three readers in the diagnosis of submucosal and perigastric varices, as well as assessments of variceal size and location. This imaging technique for detection of fundal varices does not differ substantially from imaging protocols that are currently used for MDCT angiography. The main difference is oral ingestion of plain water to distend the stomach and gastric fundus, which is essential for distinction between submucosal and perigastric fundal varices. MDCT scanners are currently widely available throughout the entire world and in our experience reading of these cases does not require a long learning curve.

Compared with EUS, a high correlation was obtained by all three independent readers who evaluated the MDCT angiograms for the presence of submucosal and perigastric fundal varices. Only in one patient was there a disagreement between EUS and MDCT angiography in the detection of a small $(<5$ $\mathrm{mm}$ in diameter) submucosal fundal varix. It is of interest to emphasise that based on MDCT angiography, all three readers noted that a submucosal varix was probably or definitively present (scores 4 and 5). In a repeated EUS, which was performed later and which was not part of the study protocol, the presence of a submucosal varix was confirmed in this patient. Hence it may be hypothesised that the diagnostic performance of MDCT angiography in the detection of submucosal fundal varices exceeds that of EUS. However, this hypothesis needs to be validated in a larger group of patients.

In this study, we demonstrated that MDCT angiography was capable of differentiating between submucosal and perigastric fundal varices which is of paramount clinical interest. MDCT angiography may also be useful for assessment of the therapeutic effect of endoscopic sclerotherapy of fundal varices. In our series, MDCT angiography demonstrated residual submucosal fundal varices in all patients who had a history of prior endoscopic sclerotherapy. In addition, MDCT angiography allows identification of the afferent and efferent veins of fundal varices. This may be of clinical and therapeutic relevance with regard to planning of alternative therapeutic strategies such as balloon occluded retrograde transvenous obliteration of gastric varices. ${ }^{28}$ 
An important drawback of MDCT angiography for imaging fundal varices is the applied radiation dose inherent in this technique. In our study, an effective dose of 4-5 mSv was calculated for both men and women. In addition, intravenous application of iodinated contrast medium may be contraindicated in patients with renal insufficiency. This is even more important in patients with end stage liver cirrhosis as those patients often suffer from additional renal insufficiency. ${ }^{29}$ Furthermore, treatment of fundal varices cannot be followed immediately in the same session after diagnosis of submucosal fundal varices on MDCT angiography. These drawbacks may hamper its routine use in patients with suspected fundal varices and may justify its indication in clinical situations when EUS may be not available or is contradicted (for example, in patients with large oesophageal varices or after ligation therapy of oesophageal varices).

The following limitations of the study need to be addressed. As only patients with suspected fundal varices on oesophagogastroduodenoscopy and no control group were included in our study, this may have resulted in inclusion bias. Another important limitation is the lack of a true diagnostic standard of reference for the presence of submucosal and perigastric fundal varices, such as a pathological correlation in all patients. In addition, a small number of patients were included in our study group. Clearly, more data are needed to determine if MDCT angiography is useful as a screening tool for detection of submucosal fundal varices in patients with portal hypertension. Finally, we did not evaluate the impact of MDCT angiography on treatment of gastric fundal varices. The optimal therapy of bleeding gastric fundal varices (for example, sclerosing therapy, ligation therapy, TIPSS, balloon occluded retrograde transvenous obliteration, or surgery) as well as the question of prevention and the effect of oesophageal variceal eradication on gastric fundal varices have not yet been determined. ${ }^{24}$ Future prospective studies are warranted to evaluate whether diagnosis and classification of submucosal fundal varices by MDCT angiography influences the decision making of gastroenterologists treating patients with gastric fundal varices.

In conclusion, this study has demonstrated for the first time that MDCT angiography is equivalent to EUS in the detection and characterisation of fundal varices, in particular with regard to the distinction between submucosal and perigastric fundal varices. MDCT angiography may become an important supplemental or alternative technique in clinical situations when EUS is not available or is contradicted.

\section{ACKNOWLEDGEMENTS}

The authors thank Peter Roth for drawing figure 1.

\section{Authors' affiliations}

J K Willmann, D Weishaupt, T Böhm, T Pfammatter, B Marincek, Institute of Diagnostic Radiology, University Hospital Zurich, Zurich, Switzerland

B Seifert, Department of Biostatistics, University of Zurich, Zurich, Switzerland

P Bauerfeind, Division of Gastroenterology, University Hospital Zurich, Zurich, Switzerland

\section{REFERENCES}

1 Sarin SK, Lahoti D, Saxena SP, et al. Prevalence, classification and natural history of gastric varices: a long-term follow-up study in 568 portal hypertension patients. Hepatology 1992;16:1343-9.

2 Rockey DC. Management of gastric varices. Gastroenterology $2001 ; 120: 1875-6$.

3 Hashizume M, Kitano S, Sugimachi K, et al. Three-dimensional view of the vascular structure of the lower esophagus in clinical portal hypertension. Hepatology 1988;8:1482-7.

4 Kimura K, Ohto M, Matsutani S, et al. Relative frequencies of portosystemic pathways and renal shunt formation through the "posterior" gastric vein: portographic study in 460 patients. Hepatology $1990 \cdot 12 \cdot 725-8$.

5 Boustiere C, Dumas O, Jouffre C, et al. Endoscopic ultrasonography classification of gastric varices in patients with cirrhosis. Comparison with endoscopic findings. J Hepatol 1993; 19:268-72.

6 Liu JB, Miller LS, Feld RI, et al. Gastric and esophageal varices: $20-\mathrm{MHz}$ transnasal endoluminal US. Radiology 1993;187:363-6.

7 Caletti GC, Brocchi E, Ferrari A, et al. Value of endoscopic ultrasonography in the management of portal hypertension. Endoscopy 1992;24(suppl 1:)342-6.

8 Sanyal AJ. The value of EUS in the management of portal hypertension. Gastrointest Endosc 2000;52:575-7.

9 Hosking SW, Johnson AG. Gastric varices: a proposed classification leading to management. BrJ Surg 1988;75: 195-6.

10 Boyce GA, Sivak MV Jr, Rosch T, et al. Evaluation of submucosal upper gastrointestinal tract lesions by endoscopic ultrasound. Gastrointest Endosc 1991;37:449-54.

11 Marn CS, Glazer GM, Williams DM, et al. CT-angiographic correlation of collateral venous pathways in isolated splenic vein occlusion: new observations. Radiology 1990;175:375-80.

12 Cho KC, Patel YD, Wachsberg RH, et al. Varices in portal hypertension: evaluation with CT. Radiographics 1995;15:609-22.

13 Ito K, Blasbalg R, Hussain SM, et al. Portal vein and its tributaries: evaluation with thin-section three-dimensional contrast-enhanced dynamic fat-suppressed MR imaging. Radiology 2000;215:381-6.

14 Muhletaler C, Gerlock AJ Jr, Goncharenko V, et al. Gastric varices secondary to splenic vein occlusion: radiographic diagnosis and clinical significance. Radiology 1979;132:593-8.

15 Horton KM, Fishman EK. 3D CT angiography of the celiac and superior mesenteric arteries with multidetector $\mathrm{CT}$ data sets: preliminary observations. Abdom Imaging 2000;25:523-5.

16 Matsumoto A, Kitamoto $M$, Imamura $M$, et al. Three-dimensional portography using multislice helical CT is clinically useful for management of gastric fundic varices. AJR Am J Roentgenol 2001; 176:899-905.

17 Takahashi S, Murakami T, Takamura M, et al. Multi-detector row helical CT angiography of hepatic vessels: depiction with dual-arterial phase acquisition during single breath hold. Radiology 2002;222:81-8.

18 Sarin SK, Kumar A Gastric varices: profile, classification, and management. Am J Gastroenterol 1989;84:1244-9.

19 Balthazar EJ, Megibow A, Naidich D, et al. Computed tomographic recognition of gastric varices. AJR Am J Roentgenol 1984;142:1 $121-5$.

20 Faigel DO, Rosen HR, Sasaki A, et al. EUS in cirrhotic patients with and without prior variceal hemorrhage in comparison with noncirrhotic control subjects. Gastrointest Endosc 2000;52:455-62.

21 Leung VK, Sung JJ, Ahuja AT, et al. Large paraesophageal varices on endosonography predict recurrence of esophageal varices and rebleeding. Gastroenterology 1997;112:1811-16.

22 Burtin P, Cales P, Oberti F, et al. Endoscopic ultrasonographic signs of portal hypertension in cirrhosis. Gastrointest Endosc 1996;44:257-61.

23 Landis JR, Koch GG. The measurement of observer agreement for categorical data. Biometrics 1977;33:159-74.

24 Blendis L, Wong F. Stopping the unstoppable? Gastroenterology 2000;118:1270-2.

25 Gress F, Schmitt C, Savides T, et al. Interobserver agreement for EUS in the evaluation and diagnosis of submucosal masses. Gastrointest Endosc 2001;53:71-6.

26 Pfau PR, Chak A. Endoscopic ultrasonography. Endoscopy 2002;34:21-8.

27 Marshall JP II , Smith PD, Hoyumpa AM Jr. Gastric varices. Problem in diagnosis. Am J Dig Dis 1977;22:947-55.

28 Ibukuro K, Mori K, Tsukiyama T, et al. Balloon-occluded retrograde transvenous obliteration of gastric varix draining via the left inferior phrenic vein into the left hepatic vein. Cardiovasc Intervent Radiol 1999:22:415-17.

29 Arroyo V, Guevara M, Gines P. Hepatorenal syndrome in cirrhosis: pathogenesis and treatment. Gastroenterology 2002;122:1658-76. 